\title{
Evaluating the fall risk among elderly population by choice step reaction test
}

This article was published in the following Dove Press journal:

Clinical Interventions in Aging

10 August 2016

Number of times this article has been viewed

\section{Donghai Wang' \\ Jian Zhang' \\ Yuliang Sun ${ }^{2}$ \\ Wenfei Zhu ${ }^{2}$ \\ Shiliu Tian' \\ Yu Liu'}

'Key Laboratory of Exercise and Health Sciences of the Ministry of Education, Shanghai University of Sport, Shanghai, People's Republic of China; ${ }^{2}$ School of Physical

Education, Shaanxi Normal University,

Xian, People's Republic of China
Correspondence: Yu Liu

Key Laboratory of Exercise and Health

Sciences of the Ministry of Education,

Shanghai University of Sport, 650 Qing

Yuan Huan Road, Shanghai 200438,

People's Republic of China

Tel +86 I35 24752 I 85

Email yuliu@sus.edu.cn
Abstract: Falls during daily activities are often associated with injuries and physical disabilities, thereby affecting quality of life among elder adults. Balance control, which is crucial in avoiding falls, is composed of two elements: muscle strength and central nervous system (CNS) control. A number of studies have reported that reduced muscle strength raises the risk of falling. However, to date there has been only limited research focused on the relationship between fall risk and the CNS. This study aimed to investigate the relationship between CNS and risk of falling among the elderly. A total of 140 elderly people (92 females and 48 males) were divided into faller and nonfaller groups based on questionnaire responses concerning falls in their daily life. Participants undertook a choice step reaction test in which they were required to respond to random visual stimuli using foot movements as fast as possible in the left or right directions. Response time was quantified as premotor time (PMT) and motor time (MT). In addition, the participants' electromyography data were recorded during the choice step reaction test. A maximal isokinetic torque test was also performed. PMT was greater in the fallers than in the nonfallers group. There was a significant difference between fall status and direction on PMT. PMT of the left limb in nonfallers was faster than the right, but in fallers there was no difference between left and right limbs. A similar phenomenon was also observed for MT. There were significant differences between fallers and nonfallers in maximum isokinetic torque at knee and ankle joints. The correct rate of PMT was higher than other variables, such as MT and maximal isokinetic torque, in evaluating elderly fall risk by using logistic regression analyses. The results suggest that PMT in the choice step reaction test could be a useful parameter to assess risk of fall among elder adults. In addition, decreased maximal isokinetic torque was related to greater PMT and disappearance of asymmetry in older adults who were at higher risk of fall, especially in the lower limb.

Keywords: falling, elderly, choice step reaction test, premotor time

\section{Introduction}

Falls during daily activities can lead to injuries and physical disabilities among older adults, thereby affecting their quality of life. ${ }^{1}$ Since the 1990 s, falls in older adults have been a major health concern in Asian countries. ${ }^{2,3}$ The consequences of falls are known to contribute substantially to the prevalence of many health problems ${ }^{4}$ and increased health care cost. ${ }^{5}$ Prevention of falls is, therefore, of great public health concern, and it is important for fall prevention to address people at risk. ${ }^{6}$

Age-related changes in the musculoskeletal system and central nervous system (CNS), which are believed to be associated with fall risk, have been widely investigated. ${ }^{4,7}$ Reduced muscle strength has been found to be one of the most important risk factors for falls. ${ }^{8-13}$ In literature, researchers have reported relationships demonstrating the contribution of lower limb muscle strength, ${ }^{14}$ such as leg press push-off strength, ${ }^{11}$ to fall incidences. For example, Berg et $\mathrm{al}^{15}$ found the maximal isokinetic 
torque of the lower limb accounted for up to $60 \%$ of falls in older adults when stepping over an obstacle, suggesting that age-related impairment in the musculoskeletal system could lead to an increased risk of falling. Therefore, lower limb muscle strength may be a sensitive index to evaluate fall risk among older adults.

However, as a failure in postural control, falls are associated with not only muscle strength, but also central neural control processes. ${ }^{16-19}$ Choice step reaction time (CSRT) is the reaction time (RT) for a task in which a subject has to make one or two or more choices and make a physical response. ${ }^{20}$ It is one of the important neural control variables and involves complicated motor responses such as extending and flexing the hip, knee, and ankle. ${ }^{21,22}$ Typically, CSRT requires participants to respond to an unpredictable stimulus as fast as possible by stepping on a designated spot according to the stimulus location. Therefore, CSRT reflects response speed and quality of motor behavior, which means CSRT is a synthesized index that can be used to evaluate a participant's function of neural control process.

As the function of the central neural system declines with age, CNS function is generally more of an issue with older adults than with the young. ${ }^{23}$ Previous studies ${ }^{22,24}$ have investigated the determinants of CSRT in evaluating fall risks among the elderly. They found that elderly participants who were in the high fall risk group had longer CSRT than those in the low fall risk group, and the longer CSRT was associated with increases in both initiation and weight transfer times. In another study, Lord and Fitzpatrick ${ }^{25}$ found that impaired quadriceps strength was one of the best predictors for increased CSRTs, and participants with a history of falls had significantly greater CSRTs compared with nonfallers. The authors concluded that increased CSRT was significantly associated with the risk of falls.

CSRT consists of two parts for two different motor control process: pre-motor time (PMT) for visual-motor process, and motor time (MT) for peripheral motor processes. ${ }^{26}$ In executing a response to the presence of unpredictable stimuli, PMT reflects the process of perception and decision making, while MT describes the function of musculoskeletal system in response to an unpredictable stimuli. Understanding PMT and MT would enhance our understanding of motor controls lower extremity movements. ${ }^{20}$ A previous study suggests that MT reflects the ability to execute movement. ${ }^{27}$ However, our previous study ${ }^{28}$ indicates that PMT may play a more important role than MT in movement execution. The results of our previous study showed that there was no significant difference between athletes and nonathlete university students in MT, but there was a significant difference in PMT. We also observed a high correlation between PMT and RT (ranging from 0.87 to 0.91 ), but not between MT and RT (ranging from 0.13 to 0.34 ). These findings suggest that PMT may be more important than MT in response to unpredictable stimuli.

Our recent review of literature found obvious limitations in studies on evaluating risk of falls among older adults indicating the need of studies on PMT and MT of individuals at risk of falls. Most of studies on the risk factors of falls were focused on the relationship between fall risks and muscle strength. Although some studies investigated the relationship between CSRT and risk of falls for old adults, ${ }^{10,14,19,23,29}$ the relationships of PMT and MT with the risk of falls have not been studied. As previous studies indicated, PMT is determined not only by the function of the central nervous system, but also by muscle strength. ${ }^{16,17,26}$ PMT, therefore, may be more closely related to fall risks among older adults than the CSRT and MT, and may provide more information related to the risk of falls of old adults.

Therefore, the purpose of this study was to investigate the relationship between PMT and MT in CSRT, maximal isokinetic torque of the lower limb, and risk of falls among elderly. We hypothesized that PMT, MT, and maximal isokinetic strengths of knee and ankle joints were significantly different between fallers and non-fallers. We also hypothesized that PMT and MT were significantly affected by the movement directions. We further hypothesized that fallers would be predicted by PMT and MT.

\section{Methods}

\section{Participants}

Initially, a total of 406 older adults between 55 and 75 years of age, living in the Guohe community, Shanghai, People's Republic of China, were contacted for eligibility screening by flyers posted in local community centers and hospitals. All the potential participants received a comprehensive explanation of the proposed study, its benefits, potential risks, and expected time commitment for participation. The participants were recruited if their scores on the activities of daily living table and International Physical Activity Questionnaire were moderate and dependent. ${ }^{25}$ Participants were excluded if they reported any of the following conditions: neurological diseases that impair mobility, dementia, visual deficits, and cardiovascular disease symptomatic during moderate exercise, poorly controlled hypertension, and use of specific medications known to impair balance. ${ }^{30}$

A total of 140 healthy older adults (92 females and 48 males) met the criteria and completed the measurements. A flowchart detailing the recruitment process is shown in Figure 1, and basic information of sample is listed 


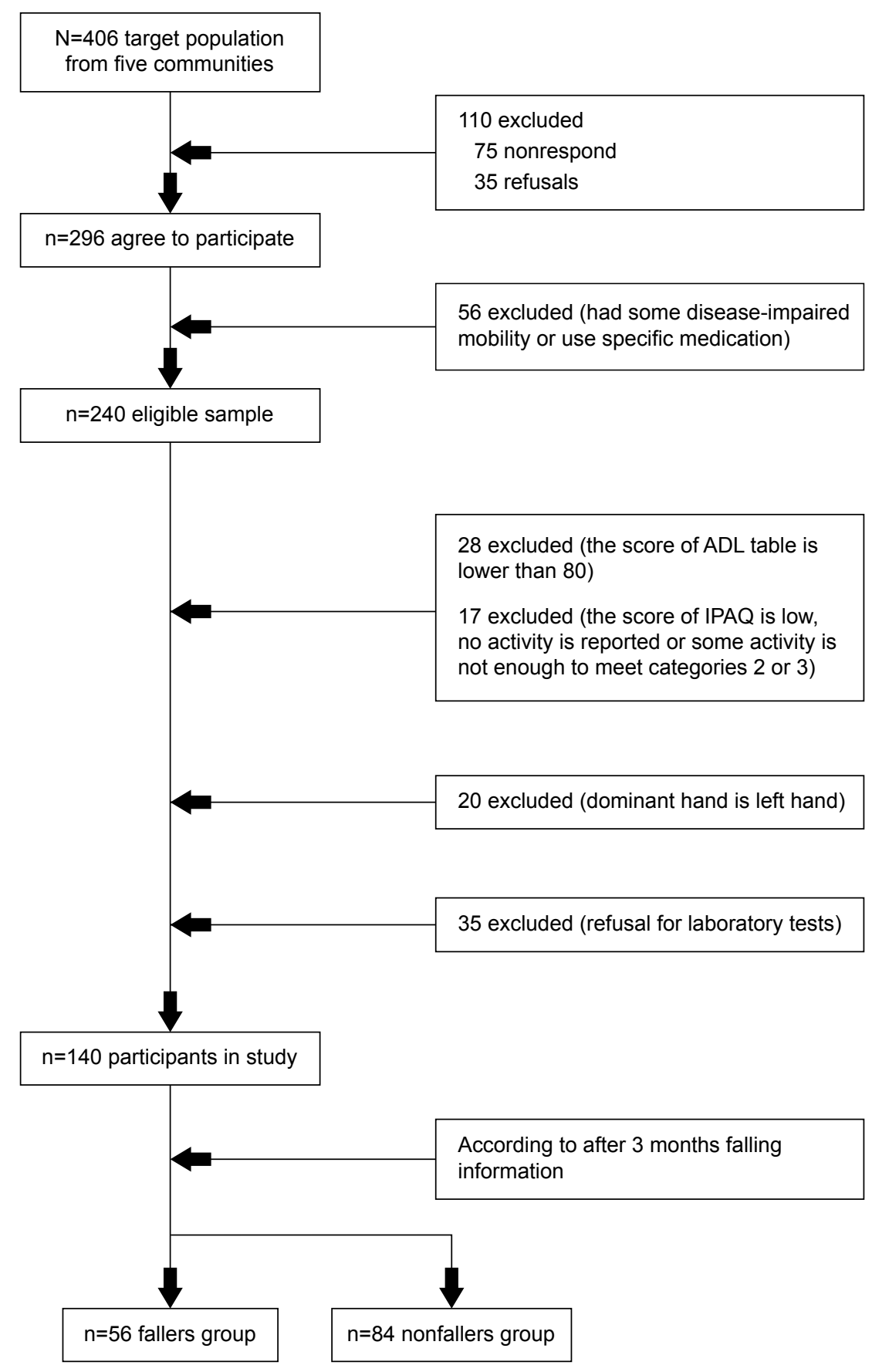

Figure I Flowchart of subjects through the study.

Abbreviations: ADL, activities of daily living; IPAQ, International Physical Activity Questionnaire.

in Table 1. According to the participants' responses to the fall questionnaire, ${ }^{31}$ we divided them into faller group $(\mathrm{n}=56$, age $=69.8 \pm 5.39$ years, height $=159.75 \pm 7.56 \mathrm{~cm}$, weight $=60.98 \pm 9.48 \mathrm{~kg})$ and nonfaller group $(\mathrm{n}=84$, age $=70.2 \pm 4.68$ years, height $=158.68 \pm 6.89 \mathrm{~cm}$, weight $=61.28 \pm 8.68 \mathrm{~kg}$ ). A participant was categorized as a faller if he or she experienced a fall within the 3-month period after the tests, ${ }^{31}$ with a fall being defined as an unexpected event in which the participant comes to rest on the ground, floor, or lower level due to any causes, ${ }^{32}$ according to the Prevention of Falls Network Europe. All participants were right-foot dominant. ${ }^{33}$ All participants were followed up successfully after 3 months. This study was approved by the Human Ethics Committee of Shanghai University of Sport. All participants provided a written consent prior to the study.

\section{Protocol}

All the tests were completed in Shanghai University of Sport, Sports Performance Research Centre (Shanghai, People's 
Table I Basic information of the participants

\begin{tabular}{lll}
\hline Group & $\begin{array}{l}\text { Fallers group } \\
(\mathbf{n = 5 6 )}\end{array}$ & $\begin{array}{l}\text { Nonfallers } \\
\text { group }(\mathbf{n = 8 4})\end{array}$ \\
\hline Age (year) & $69.8 \pm 5.4$ & $70.2 \pm 4.7$ \\
Number of female & 32 & 60 \\
Number of male & 24 & 24 \\
Height $(\mathrm{cm})$ & $159.8 \pm 7.6$ & $158.7 \pm 6.9$ \\
Body mass $(\mathrm{kg})$ & $61.0 \pm 9.5$ & $61.3 \pm 8.7$ \\
Length of lower limb $(\mathrm{cm})$ & $75.5 \pm 3.5$ & $74.6 \pm 2.3$ \\
ADL score & $85.2 \pm 4.4$ & $84.6 \pm 2.7$ \\
IPAQ score & Moderate & Moderate \\
\hline
\end{tabular}

Notes: Data presented as mean \pm standard deviation unless stated otherwise. The criteria of ADL score: 100 (independence), 75-95 (mild dependence), 50-70 (moderate dependence), 25-45 (heavy dependence), and 0-20 (total dependence). IPAQ2: categorical score - three levels of physical activity are proposed: I) low (no activity is reported OR, some activity is reported but not enough to meet Categories 2 or 3). 2) Moderate (either of the following three criteria, $\geq 3$ or more days of vigorous activity of at least 20 minutes per day $O R, \geq 5$ or more days of moderate-intensity activity and/or walking of at least 30 minutes per day, or $\geq 5$ or more days of any combination of walking, moderate-intensity or vigorous-intensity activities achieving a minimum of at least $600 \mathrm{MET}-\mathrm{min} / \mathrm{wk}$ ). 3) High (any one of the following two criteria, vigorous-intensity activity on at least 3 days and accumulating at least 3 days and accumulating at least I,500 MET-min/wk OR $\geq 7$ or more days of any combination of walking, moderate- or vigorous-intensity activities accumulating at least 3,000 MET-min/wk).

Abbreviations: $A D L$, activities of daily living; IPAQ, International Physical Activity Questionnaire; OR, odd ratio; MET, metabolic equivalent of energy.

Republic of China). The eight testers in this study were graduate students who majored in biomechanics and had at least 1 year of training with the equipment and techniques used in this study.

Each participant had a CSRT. In CSRT, the participant was asked to stand in front of a stimulus device that had three lightemitting diodes (LEDs) displayed at eye level (Figure 2). The central LED (red) served as fixation point and the two outer

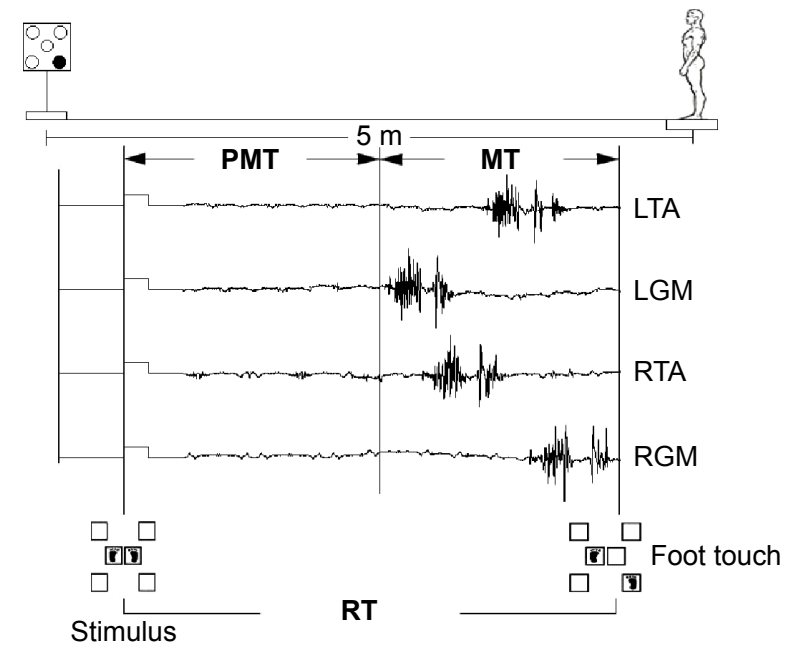

Figure 2 Sketch of the experimental setup and illustration of the EMG and foot touch data used to determine PMT and MT in the CSRT.

Abbreviations: RT, response time; EMG, electromyography; PMT, premotor time; MT, motor time; CSRT, choice step reaction time; LTA, left tibialis anterior; LGM, left gastrocnemius medial; RTA, right tibialis anterior; RGM, right gastrocnemius medial. ones (green) were stimuli. The two outer LEDs were placed on the left and right sides. The participants were instructed to respond to the stimulus as fast as possible by stepping on a designated spot according to the stimulus location. A $5 \mathrm{~m}$ gap was placed between the signal light and the participant, and there was a $30 \mathrm{~cm}$ gap between each signal light.

The left and right direction signal LEDs lit up randomly. The interval time was $1,500,2,000$, or 2,500 milliseconds. ${ }^{28}$ The participants could not predict the direction or time of appearance of the signal. Before testing, the participants had one training session of three trials on each of the two directions. The test itself consisted of two blocks of 25 trials for a total of 50 response trials. There was a 2-minute rest between two blocks. The test was performed separately for the right and left legs.

During each CSRT, the participant's lower limb muscle electromyography $(\mathrm{EMG})$ data $(1,000 \mathrm{~Hz})$ were recorded via a 16-channel analog systems (Biovision, Heidelberg, German). More specifically, $\mathrm{Ag} / \mathrm{AgCl}$ bipolar surface EMG electrodes $(1.1 \mathrm{~cm}$ diameter and $3.5 \mathrm{~cm}$ interelectrode distance) were placed over the muscle bellies and aligned with the muscle fibers according to the published guidelines ${ }^{28}$ to collect EMG data for the following four muscles: tibialis anterior (TA) and gastrocnemius medial (GM) of right and left limbs.

\section{Maximal isokinetics torque test}

Maximal isokinetic torque was assessed with a Con-trex isokinetic dynamometer (Con-trex Corporation, Zurich, Switzerland). Tests were conducted to assess the maximal isokinetic torque of the right knee and ankle extensors and flexors separately at angular velocities of $60^{\circ} / \mathrm{s} .{ }^{14}$ The maximal isokinetic torque was determined in one set of five maximal repetitions, and the highest peak torque was used in the data analysis. There was a 5-minute rest after each maximal isokinetic torque test to avoid possible fatigue effects. In addition, the participants were encouraged verbally to exert as much force as possible.

\section{Data reduction}

Raw EMG data were filtered through a band-pass digital filter at a high-pass cutoff frequency of $10 \mathrm{~Hz}$ and a low-pass cutoff frequency of $400 \mathrm{~Hz}$, full-wave rectified, and then smoothed with a second order recursive Butterworth low-pass digital filter at $6 \mathrm{~Hz}$ to obtain linear envelop EMG. All EMG data processing were performed using an EMG-specific Wavelet Analysis computer program package (DASYLab, National Instruments, USA). CSRT was calculated as the time when 
the foot step on the target minus the time of the presentation of the stimulus. The onset time of each muscle was identified from linear envelop EMG. The onset time of a muscle was defined as the beginning time of the first burst of linear envelop EMG greater than three standard deviations above baseline envelop EMG for more than 25 ms. ${ }^{34}$ PMT was calculated as earliest muscle onset time minus the onset time of the stimulus. MT was calculated as CSRT minus PMT. A trial with a PMT shorter than $100 \mathrm{~ms}$ was considered as an anticipated response and excluded from data analysis. ${ }^{35,36}$ Trials in which responses made in an incorrect direction were also excluded. The estimated maximum overt error rate was $6 \% .{ }^{28}$ The six trials with the shortest CSRTs in left and right directions were identified for each participant and included in statistical analysis.

Peak isokinetic torque in each isokinetic strength testing trial was identified. The maximum of the five peak isokinetic torques in each joint movement direction was used to represent the maximal isokinetic strength of the given joint of the participant in the given joint movement direction.

\section{Data analyses}

Descriptive statistical analysis was conducted for all variables whenever appropriate (eg, mean values, standard deviations, frequencies). A two-factor mixed model repeated measures Multivariate analysis of variance was used to examine the association of fall status (nonfallers and fallers) and directions (left and right) with PMT and MT. The maximal isokinetic torque was analyzed by an independent samples $t$-test to examine the difference between nonfallers and fallers. A Type I error rate less than or equal to 0.05 was chosen as the indication of statistical significance. A Bonferroni adjustment was made to adjust the Type I error rate as an indication of statistical significance to 0.025 for $t$-tests to guarantee the overall Type I error rate was no greater than 0.05 .

The probability of falls was predicted as functions of PMT, MT, and maximal isometric torques of knee and ankle joints, respectively, through logistic regression analyses. Receiver operating curve analysis was performed for each regression analysis to determine the cutoff magnitude of the given predictor for falls as a definition of fallers. The percentage of correct prediction of each definition of falls was calculated, and used as the sensitivity measure of the predictor. All data analyses were performed using the SPSS statistical package, version 20.0 (IBM Corporation, Armonk, NY, USA).

\section{Results \\ Choice reaction tests}

Table 2 details PMT and MT by fall status and direction. PMT of lower limbs was significantly greater in fallers than in nonfallers $(456.08 \pm 52.44$ vs $387.72 \pm 52.44$ milliseconds respectively, $\left.F_{1,138}=229.98, P<0.001\right)$. PMT was associated with left and right side (405.55 \pm 52.54 vs $424.58 \pm 51.89$ milliseconds respectively, $\left.F_{1,138}=10.608, P<0.001\right)$. We also observed a significant interaction between fall status and direction on PMT $\left(F_{1,138}=4.52, P=0.035\right)$. PMT of the left limb in nonfallers was faster than the right $(\mathrm{PMT}=373.82 \pm 38.34$ vs $401.62 \pm 52.44$ milliseconds, respectively), but in fallers there was no difference between left and right (453.15 \pm 30.21 vs $459.00 \pm 25.37$ milliseconds).

There was a significant group difference in MT $\left(F_{1,138}=342.26, P<0.001\right)$ between fallers and nonfallers, with the nonfaller group (731.01 \pm 44.86 milliseconds) being faster than the faller group (807.21 \pm 36.02 milliseconds). There was also a significant laterality difference in MT $\left(F_{1,138}=50.510, P<0.001\right)$, and the left sides $(742.99 \pm 60.23$ milliseconds) being faster than right sides $(780.01 \pm 44.079$ milliseconds) when responding to a stimulus signal light. We also observed a significant interaction between fall status and directions $\left(F_{1,138}=16.64, P<0.001\right)$ on MT. There was no difference in fallers' MT between left and right sides (800.14 \pm 39.88 vs $814.29 \pm 30.42$ milliseconds, $P=0.037$ ), but in nonfallers, the left side (704.88 \pm 36.72 milliseconds) was faster than the right side (757.14 \pm 36.31 milliseconds).

\section{Maximum isokinetic torque}

Table 3 lists the maximal isokinetic torque of knee and ankle joint. Maximum isokinetic torque of the knee flexor was

Table 2 PMT and MT on left and right directions between fallers and nonfallers group (mean \pm standard deviation)

\begin{tabular}{|c|c|c|c|c|c|c|}
\hline \multirow[t]{2}{*}{ Variables } & \multicolumn{2}{|l|}{ Left } & \multicolumn{2}{|l|}{ Right } & \multicolumn{2}{|c|}{ Group direction* } \\
\hline & Nonfallers & Fallers & Nonfallers & Fallers & $F_{1,138}$ & $P$-value \\
\hline PMT (ms) & $373.82 \pm 38.34^{* * * *}$ & $453.15 \pm 30.21$ & $401.62 \pm 52.44 *$ & $459.00 \pm 25.37$ & 4.516 & 0.035 \\
\hline MT (ms) & $704.88 \pm 36.72 *, * *$ & $800.14 \pm 39.88$ & $757 .|4 \pm 36.3| *$ & $8 \mid 4.29 \pm 30.42$ & 16.644 & $<0.001$ \\
\hline
\end{tabular}

Notes: There were 56 participants in the fallers group and 84 participants in fallers group. $\alpha$ was set at 0.05 . $* P<0.01 ; * * P<0.05$. In a two-factor mixed model repeated measures MANOVA for group $\times$ direction interaction, $\alpha$ was set at 0.05 .

Abbreviations: PMT, premotor time; MT, motor time; MANOVA, Multivariate analysis of variance. 
Table 3 Maximal isokinetic torque of knee and ankle between fallers and nonfallers group (mean \pm standard deviation)

\begin{tabular}{llll}
\hline Variables & $\begin{array}{l}\text { Fallers } \\
(\mathbf{N}=\mathbf{5 6})\end{array}$ & $\begin{array}{l}\text { Nonfallers } \\
(\mathbf{N}=\mathbf{8 4})\end{array}$ & P-value \\
\hline Knee flexor $(\mathrm{Nm})$ & $30.3 \pm 7.60$ & $40.91 \pm 8.44$ & $<0.00 \mathrm{I}$ \\
Knee extensor $(\mathrm{Nm})$ & $53.20 \pm 10.85$ & $67.98 \pm 17.68$ & $<0.00 \mathrm{I}$ \\
Ankle dorsiflexor $(\mathrm{Nm})$ & $34.27 \pm 7.17$ & $40.81 \pm 6.96$ & $<0.00 \mathrm{I}$ \\
Ankle plantar flexor $(\mathrm{Nm})$ & $56.39 \pm 11.83$ & $65.51 \pm 12.15$ & $<0.00 \mathrm{I}$ \\
\hline
\end{tabular}

significantly greater in nonfallers than in fallers $(40.91 \pm 8.44$ vs $30.30 \pm 7.60 \mathrm{Nm}$, respectively, $P<0.001)$. Maximum isokinetic torque of knee extensor in the nonfallers group $(67.98 \pm 17.68 \mathrm{Nm})$ was also greater than that in fallers group (53.20 $\pm 10.85 \mathrm{Nm}, P<0.001)$.

Independent $t$-tests showed that the maximum isokinetic knee flexion and extension torques were significantly greater in non-fallers compared to fallers $(P=0.001$ and $P=0.001)$ (Table 3). Independent $t$-tests also showed that the maximal isokinetic ankle dorsiflexion and plantarflexion torques were significantly greater in non-fallers compared to fallers $(P=0.001$ and $P=0.001)$.

\section{Logistic regression results on PMT, MT, and maximal isokinetic torque}

With fallers defined as PMT and MT greater than 425.2 and $764.9 \mathrm{~ms}$, respectively, PMT and MT predicted fallers with $92.1 \%$ and $82.1 \%$ accuracies, respectively (Table 4 ). With fallers defined as maximal knee flexion and extension torques and ankle dorsiflxion and planarflexion torques less than $32.5,55.0,34.8$, and $55.8 \mathrm{Nm}$, respectively, these variables predicted fallers with $82.1 \%, 70.7 \%, 80.0 \%$, and $75.7 \%$ accuracies, respectively (Table 4).

\section{Discussion}

The purpose of this study was to determine the relationships of the risk of falls with the PMT and MT determined from CSR test and lower limb maximal isokinetic torque in older adults. We hypothesized that PMT was more closely correlated with fall risk, and was more accurate in predicting fall risk among the elderly than maximal isokinetic torque in the lower limb or MT.

The PMT of the nonfallers was faster than the fallers ( $\mathrm{PMT}=387.72 \pm 47.87$ vs $456.07 \pm 27.92$ milliseconds, respectively). In a previous study, Lord and Fitzpatrick ${ }^{25}$ used CSRT measurements to estimate risk of falls. They found that the CSRT in older adults was greater than that in young people, and this phenomenon was due to the fact that muscle strength declined significantly in older adults. To our knowledge, no studies have explored whether PMT is sensitive in evaluating falling risk. In this study, we divided CSRT into PMT and MT and compared them between faller and nonfaller groups. The results of our study provided strong evidence that high PMT in older adults is associated with higher risk of falling. Prevention of falls depends upon the timely initiation of an appropriate postural response ${ }^{7}$ and, although situations jeopardizing balance occur in all age groups, postural adjustments in older adults are characterized by the absence of speed and adroitness that is necessary to ensure the recovery of stability. ${ }^{29,37}$ PMT can be considered as a variable that reflects older adults' neuropsychological, sensorimotor, and balance ability in the initiation of fast and appropriate step responses. Consequently, PMT plays an important role in evaluating older adults' fall risk.

The fallers had greater MT than nonfallers $(807.21 \pm 36.01$ vs $731.01 \pm 36.31$ milliseconds, respectively). A previous study $^{38}$ concluded that older adults whose lower muscle strength was weak were at high risk of falling. The finding that fallers also have greater MT, after controlling for lower limb maximal isokinetic torque, suggests an additional explanation for the higher falling risk in fallers group.

The results of the maximal isokinetic torque measurements at knee and ankle showed that nonfallers had significantly greater maximal isokinetic torque than fallers. Previous studies have found that the decline in the speed of motor organization and action planning had a high correlation with the decrease in muscle strength. ${ }^{7,39} \mathrm{~A}$ decline in maximal isokinetic torque will increase motor organization

Table 4 Associations of PMT, MT, and isokinetic strength with fall status

\begin{tabular}{|c|c|c|c|c|c|}
\hline Variable & $\begin{array}{l}\text { Predict } \\
\text { value }\end{array}$ & $\begin{array}{l}\text { Predict } \\
\text { accuracy (\%) }\end{array}$ & SD & OR $(95 \% \mathrm{CI})$ & $P$-value \\
\hline PMT (ms) & 425.22 & 92.1 & 40.66 & 1.227 (1.082-1.389) & $<0.001$ \\
\hline $\mathrm{MT}(\mathrm{ms})$ & 764.88 & 82.1 & 44.38 & $1.058(1.038-1.074)$ & 0.002 \\
\hline Knee flexor (Nm) & 32.46 & 82.1 & 7.60 & $0.679(0.592-0.778)$ & $<0.001$ \\
\hline Knee extensor $(\mathrm{Nm})$ & 55.01 & 70.7 & 12.45 & $0.863(0.822-0.907)$ & $<0.001$ \\
\hline Ankle dorsiflexor $(\mathrm{Nm})$ & 34.76 & 80.0 & 7.17 & $0.7 I I(0.633-0.80 I)$ & $<0.001$ \\
\hline Ankle plantar (Nm) & 55.75 & 75.7 & 9.75 & $0.857(0.810-0.906)$ & $<0.001$ \\
\hline
\end{tabular}

Abbreviations: $\mathrm{Cl}$, confidence interval; MT, motor time; PMT, premotor time; SD, standard deviation; OR, odd ratio. 
and action planning time, slowing dynamic postural adjustments and the attainment of balance. ${ }^{7,11,39}$ Friedman et $\mathrm{al}^{40}$ suggested that the decline in physical fitness makes older adults more prone to falling. During movements, their fear of falling decreases movement speed. Moreover, once fear develops, it is likely to persist and be self-reinforcing. In this study, older adults' longer PMT may be associated with their diminished motor ability, because impaired motor ability increased subjects' fear of falling and consequently prolonged motor organization and action planning. ${ }^{40}$ The meta-analysis conducted by Moreland et $\mathrm{al}^{9}$ revealed that decreased muscle strength was an important factor in falling, but did not identify the mechanism for the effect. The results of our study are consistent with the findings from previous research.

PMT was identified as a significant predictor of falls and was a more sensitive predictor than MT or maximal isokinetic torque. PMT and MT were positively correlated with fall status, whereas the maximal isokinetic torque of knee and ankle joint were negatively correlated with fall status. PMT had the highest correct prediction rate for falls (92.1\%), suggesting that PMT may be a more sensitive predictor than MT or maximal isokinetic torque. When older adults fail to control their posture and want to recover their balance, our results suggest that PMT, reflecting the decision and planning process, is a critical factor, but that MT, including both initiation and weight transfer times, is also an important factor. Complicated motor responses, such as extending and flexing the hip, knee, and ankle, are also involved in this process..$^{21,22}$ This is why the predictive accuracy of maximal isokinetic torque of knee and ankle joints is similar to MT. PMT is more sensitive to predict fall risks because it represents individuals' action planning ability, ${ }^{22,24}$ which, in turn, depends upon both CNS ability and muscle strength. ${ }^{7}$ In summary, PMT is a more comprehensive indicator and this explains why it gives the highest correct prediction rate for falls.

There were significant differences between the left and right sides in nonfallers' PMT and MT $(P<0.001)$, with PMT of the left limb being faster than the right ( $\mathrm{PMT}=373.82 \pm 38.34$ vs $401.62 \pm 52.44$ milliseconds, respectively). However, a similar phenomenon was not observed in the fallers group's PMT. During CSRT, individuals (right footed) tend to react faster using the left foot than right foot is a normal phenomenon. This phenomenon is named by asymmetry. ${ }^{33}$ The disappearance of the lateralization effect in the PMT and MT in fallers may be associated with decreased muscle strength and prolonged motor organization. Impairment of the musculoskeletal system can cause the disappearance of asymmetry and increase in risk of falling in older adult participants. Additionally, fallers' PMT in choice $\mathrm{RT}$ in the lower limb that was greater than nonfallers' was associated with a decline in fallers' muscle strength; the latter, we argue, tends to prolong motor organization and action planning. The longer PMT in the fallers may be related to deterioration in their action planning ability.

The main limitation of this study is that it is an observational study and so causal relationship cannot be concluded. Interventions, such as strength exercise or Tai Chi, are needed in future study to explore the effect of increasing maximal isokinetic torque on fall risks. In addition, the test was performed in a lab setting in this study without simulating the situation of falling to evaluate the ability to recover from losing stability of older adults. ${ }^{29,37}$ In addition, cognitive function was not measured in this study. A previous study has found the impairment of musculoskeletal system to be associated with the decline in cognitive function, ${ }^{7}$ but there are very few studies investigating the relationship between function of center nervous system and fall risk of older adults. Future studies with large sample size and including participants with different level fall risk are also needed to investigate the effect in sex or ages.

\section{Conclusion}

In conclusion, PMT in choice RT in lower limb could be a useful parameter to assess risk of fall among older adults. In addition, decreased maximal isokinetic torque was related to greater PMT and disappearance of asymmetry in older adults who were at higher risk of fall, especially in the lower limb.

\section{Acknowledgments}

This work was sponsored by the Key Laboratory of Exercise and Health Sciences of Ministry of Education, Shanghai University of Sport. This work was also supported by Innovation Cultivation Program for Graduate Student (yjscx2015017). Additional funding was supported by the Fundamental Research Funds for the Central Universities (GK201603128, GK201603129).

\section{Disclosure}

The authors report no conflicts of interests in this work.

\section{References}

1. Rubenstein LZ, Josephson KR. Falls and their prevention in elderly people: what does the evidence show? Med Clin North Am. 2006;90(5): 807-824.

2. Marcella Mun-San K, Close JC, Wong AK, Lord SR. Falls incidence, risk factors, and consequences in Chinese older people: a systematic review. J Am Geriatr Soc. 2011;59(3):536-543. 
3. Faes MC, Reelick MF, Melis RJ, Borm GF, Esselink RA, Rikkert MG. Multifactorial fall prevention for pairs of frail community-dwelling older fallers and their informal caregivers: a dead end for complex interventions in the frailest fallers. $J$ Am Med Dir Assoc. 2011;12(6):451-458.

4. Lord SR, Dayhew J. Visual risk factors for falls in older people. $J \mathrm{Am}$ Geriatr Soc. 2001;49(5):508-515.

5. Heinrich S, Rapp K, Rissmann U, Becker C, König HH. Cost of falls in old age: a systematic review. Osteoporos Int. 2009;21(6):891-902.

6. Lord SR, Castell S, Corcoran J, et al. The effect of group exercise on physical functioning and falls in frail older people living in retirement villages: a randomized, controlled trial. J Am Geriatr Soc. 2003;51(12):1685-1692.

7. Avril M, Maki BE. Are age-related impairments in change-in-support balance reactions dependent on the method of balance perturbation? J Biomech. 2009;42(8):1023-1031.

8. Bedient A, Lee SY, Jacobs K, et al. Comparisons of laboratory tests and simple clinical tests for identifying fallers among healthy older persons. Medicine \& Science in Sports \& Exercise. 2011:43(Suppl 1).

9. Moreland JD, Richardson JA, Goldsmith CH, Clase CM. Muscle weakness and falls in older adults: a systematic review and meta-analysis. J Am Geriatr Soc. 2004;52(7):1121-1129.

10. Cui Z, Dewei M, Riskowski JL, Qipeng S. Strategies of stepping over obstacles: the effects of long-term exercise in older adults. Gait Posture. 2011;34(2):191-196.

11. Boudville N, Inderjeeth C, Elder GJ, Glendenning P. Association between 25 -hydroxyvitamin $\mathrm{D}$, somatic muscle weakness and falls risk in end-stage renal failure. Clin Endocrinol. 2010;73(3):299-304.

12. Faes MC, Reelick MF, Banningh JW, Maartje De G, Esselink RA, Olde Rikkert MG. Qualitative study on the impact of falling in frail older persons and family caregivers: foundations for an intervention to prevent falls. Aging Ment Health. 2010;14(7):834-842.

13. Rapp K, Becker C, Cameron ID, König HH, Büchele G. Epidemiology of falls in residential aged care: analysis of more than 70,000 falls from residents of Bavarian nursing homes. J Am Med Dir Assoc. 2012;13(13): 187.e181-187.e186.

14. Zhuang J, Huang L, Wu Y, Zhang Y. The effectiveness of a combined exercise intervention on physical fitness factors related to falls in communitydwelling older adults. Clin Interv Aging. 2014;9(1):131-140.

15. Berg WP, Alessio HM, Mills EM, Tong C. Circumstances and consequences of falls in independent community-dwelling older adults. Age Ageing. 1997;26(4):261-268.

16. Mclean SG, Borotikar B, Lucey SM. Lower limb muscle pre-motor time measures during a choice reaction task associate with knee abduction loads during dynamic single leg landings. Clin Biomech (Bristol, Avon). 2010;25(6):563-569.

17. McIlroy WE, Maki BE. Age-related changes in compensatory stepping in response to unpredictable perturbations. J Gerontol A Biol Sci Med Sci. 1996;51(6):M289-M296.

18. Deconinck FJ, Savelsbergh GJ, De CD, Lenoir M. Balance problems during obstacle crossing in children with developmental coordination disorder. Gait Posture. 2010;32(3):327-331.

19. Spirduso WW. Reaction and movement time as a function of age and physical activity level. J Gerontol. 1975;30(4):435-440.

20. Sheppard J, Young W. Agility literature review: classifications, training and testing. J Sports Sci. 2006;24(9):919-932.

21. Lord SR, Ward JA, Williams P, Anstey KJ. Physiological factors associated with falls in older community-dwelling women. J Am Geriatr Soc. 1994;42(10):1110-1117.
22. Lord S, Clark R. Simple physiological and clinical tests for the accurate prediction of falling in older people. Gerontology. 1996;42(4): 199-203.

23. Kukulka CG, Hajela N, Olson E, Peters A, Podratz K, Quade C. Visual and cutaneous triggering of rapid step initiation. Exp Brain Res. 2009;192(2):167-173.

24. Yamada M, Higuchi T, Tanaka B, et al. Measurements of stepping accuracy in a multitarget stepping task as a potential indicator of fall risk in elderly individuals. J Gerontol. 2011;66(9):994-1000.

25. Lord SR, Fitzpatrick RC. Choice stepping reaction time a composite measure of falls risk in older people. J Gerontol A Biol Sci Med Sci. 2001;56(10):M627-M632.

26. Botwinick J, Thompson LW. Premotor and motor components of reaction time. J Exp Psychol. 1966;71(1):9.

27. Ballanger B, Boulinguez P. EMG as a key tool to assess motor lateralization and hand reaction time asymmetries. J Neurosci Methods. 2009; 179(1):85-89.

28. Zhang J, Chen R, Wu Y, et al. An EMG study on characteristics of premotor and motor components in an agility reaction time test on athletes. J Sports Med Phys Fitness. 2013;53(5):566-572.

29. Hsu WL, Chou LS, Woollacott M. Age-related changes in joint coordination during balance recovery. Age. 2013;35(4):1299-1309.

30. Borotikar BS, Newcomer R, Koppes R, McLean SG. Combined effects of fatigue and decision making on female lower limb landing postures: central and peripheral contributions to ACL injury risk. Clin Biomech. 2008;23(1):81-92.

31. Lord SR. Falls in Older People: Risk Factors and Strategies for Prevention. Cambridge, UK: Cambridge University Press; 2007.

32. Liu-Ambrose T, Ahamed Y, Graf P, Feldman F, Robinovitch SN. Older fallers with poor working memory overestimate their postural limits. Arch Phys Med Rehabil. 2008;89(7):1335-1340.

33. Olex-Zarychta D, Raczek J. The relationship of movement time to hand-foot laterality patterns. Laterality. 2008;13(5):439-455.

34. Solnik S, DeVita P, Rider P, Long B, Hortobágyi T. Teager-Kaiser Operator improves the accuracy of EMG onset detection independent of signal-to-noise ratio. Acta Bioeng Biomech. 2008;10(2):65.

35. Barthélémy S, Boulinguez P. Manual asymmetries in the directional coding of reaching: further evidence for hemispatial effects and right hemisphere dominance for movement planning. Exp Brain Res. 2002; 147(3):305-312.

36. Iacoboni M, Zaidel E. Crossed-uncrossed difference in simple reaction times to lateralized flashes: between- and within-subjects variability. Neuropsychologia. 2000;38(5):535-441.

37. Cameron MH, Lord S. Postural control in multiple sclerosis: implications for fall prevention. Curr Neurol Neurosci Rep. 2010;10(5):407-412.

38. Beyer N, Simonsen L, Bülow J, et al. Old women with a recent fall history show improved muscle strength and function sustained for 6 months after finishing training. Aging Clin Exp Res. 2007;19(4):300-309.

39. Pijnappels M, Delbaere K, Sturnieks DL, Lord SR. The association between choice stepping reaction time and falls in older adults - a path analysis model. Age Ageing. 2010;39(1):99-104.

40. Friedman SM, Munoz B, West SK, Rubin GS, Fried LP. Falls and fear of falling: which comes first? A longitudinal prediction model suggests strategies for primary and secondary prevention. J Am Geriatr Soc. 2002; 50(8):1329-1335.
Clinical Interventions in Aging

\section{Publish your work in this journal}

Clinical Interventions in Aging is an international, peer-reviewed journal focusing on evidence-based reports on the value or lack thereof of treatments intended to prevent or delay the onset of maladaptive correlates of aging in human beings. This journal is indexed on PubMed Central, MedLine,

\section{Dovepress}

CAS, Scopus and the Elsevier Bibliographic databases. The manuscript management system is completely online and includes a very quick and fair peer-review system, which is all easy to use. Visit http://www.dovepress. com/testimonials.php to read real quotes from published authors. 